\title{
Les représentations des pratiques sexuelles : du phallocentrisme au queer en passant par les réseaux sociaux
}

Christina BRASSARD, Université de Toronto

Christina CHUNG, Université de Toronto

Guillaume GIRARD, Université de Sherbrooke

En littérature, comme le souligne Cécile Laborde dans Le ravissement du style: sémiostylistique pour une réception physiologique de l'art verbal (2016), « les représentations sexuelles sont anciennes et traversent les siècles : elles sont présentes chez les Latins aussi bien que chez les Grecs, au Moyen-Âge avec les fabliaux comme à la Renaissance » (32). En parlant de l'érotisme et du pornographique, chez Baudelaire, Bataille ou Sade par exemple, Laborde met en évidence cette ampleur. Néanmoins, Pat Califia, militant des pratiques sadomasochistes et de la communauté queer, suggère dans Public Sex : The Culture of Radical Sex (1994) que les pratiques sexuelles ont longtemps été considérées comme étant indicibles et que les termes utilisés pour les décrire étaient vagues et se rapprochaient des euphémismes.

Prenant en considération ces propos, nous sommes tenté·e·s d'affirmer que les temps changent et que l'on définit désormais autrement, au moyen des représentations littéraires, cinématographiques ou artistiques, les pratiques sexuelles. Entre autres, Anne Archet, dans son recueil Le carnet écarlate. Fragments érotiques lesbiens (2014), écrit : «Elle aime juste me faire sucer ses seins en se doigtant dans la pénombre jusqu'à ce que la fatigue et la lassitude la gagnent » (103). Nul doute qu'il n'est plus étonnant aujourd'hui de lire ce genre de scènes sexuellement explicites dans les littératures. Il faut même dire qu'elles sont nombreuses. Grâce à ce dossier, nous souhaitons, tout en montrant l'actualité de cette question dans la recherche universitaire, illustrer le fait que, dans les arts et les littératures, les pratiques sexuelles sont très présentes et que les façons utilisées pour les décrire peuvent être à la fois précises et originales.

Si plusieurs études portant sur la sexualité dans les littératures et les arts ont mis l'accent sur l'aspect identitaire et discursif, peu se sont consacrées uniquement aux représentations des pratiques sexuelles, à plus forte raison en ce qui a trait à l'analyse textuelle. C'est pourquoi au printemps 2017, lors du congrès des sciences humaines à l'Université Ryerson, nous avons organisé un atelier intitulé «Représentations des pratiques sexuelles: de la modernité à la 
postmodernité », dans le but de rassembler des chercheur·e·s autour de cette question. Les discussions, fort intéressantes, nous ont amené-e·s à publier quelques-unes des communications. Notre dossier est ainsi composé de sept articles traitant des représentations des pratiques sexuelles, et ce, selon différentes approches : théories queer, études du genre sexuel et psychanalyse.

Dans le premier article, Christina Brassard analyse la manière dont Nelly Arcan désérotise des formes de pratiques sexuelles machistes et phallocentriques. Brassard s'intéresse plus précisément aux stratégies textuelles qu'utilise Arcan, une auteure qui met l'objectification féminine et la domination masculine au cœur de son œuvre. Quant à Nathanaël Wadbled, il propose une analyse de la dernière partie du film Race d'Ep de Lionel Soukaz (1979). Wadbled se concentre sur la rencontre avortée entre un personnage homosexuel français et un personnage hétérosexuel états-unien de passage à Paris. À la lumière de quelques écrits de Jacques Lacan, de Guy Hochquenghem et d'autres théoriciens, Wadbled met en exergue l'incommunicabilité entre les deux hommes et l'impossibilité pour le personnage homosexuel de vivre un réel rapport sexuel avec le personnage hétérosexuel. L'article montre que, lorsque les minorités sexuelles se contentent d'être tolérées par la société hétéronormative, il ne leur reste plus que leurs fantasmes. Dans son étude de l'œuvre de Pierre Molinier, Mélodie Marull explore le fétichisme et le travestissement. À travers la déconstruction et la reconstruction de son propre corps au moyen de la photographie, Molinier représente le genre sexuel et la sexualité de façons non-binaire et nonnormative. Pour sa part, Guillaume Girard examine l'influence des réseaux sociaux sur les comportements sexuels du narrateur de Satyriasis: (mes années romantiques) de Guillaume Lambert. Son hypothèse est que les sites et applications de rencontre sont des dispositifs de subjectivation (Agamben) qui produisent des comportements et qui s'inscrivent dans l'ère pharmacopornographique (Preciado), laquelle obéit au circuit productif excitation-frustrationexcitation (Preciado). Girard analyse dès lors le discours que Lambert tient sur les réseaux sociaux et la façon dont il les représente, notamment à travers les relations entre les personnages du roman. S'ensuit l'article de Christina Chung, dans lequel elle s'inspire des théories féministes, queer et du BDSM pour analyser la représentation des pratiques sexuelles, telles que l'ondinisme, la soumission et la domination, et le fist-fucking dans Quatrième génération et Insurrections ! en territoire sexuel de Wendy Delorme. De son côté, Enda McCaffrey étudie le film Ici commence la nuit d'Alain Guiraudie tout en mettant en relief les débats relatifs à la relationalité et la (non-) relationalité, qui animent aujourd'hui les chercheur·e·s en théories queer. Selon lui, l'originalité 
de Guiraudie se trouve dans sa manière d'exposer l'idée que la sexualité queer aurait un futur. Dans l'article qui clôt le dossier, Gabriel Rémy-Handfield se penche sur le roman de Gabrielle Wittkop, Le nécrophile, et fait ressortir la manière dont la perversion se rapproche de la théorie queer. Rémy-Handfield se base sur les théories de James Penney, de Gilles Deleuze et de Lee Edelman afin de montrer en quoi la sexualité du narrateur du roman contourne le régime hétéronormatif.

Nous espérons que ce numéro de Voix plurielles contribuera aux recherches et aux réflexions sur les pratiques sexuelles et sur leurs représentations, qu'elles soient en littérature, au cinéma ou en art. Nous tenons à remercier l'équipe éditoriale de Voix plurielles, qui nous a permis de mener à bien ce projet, de même que les évaluateurs et évaluatrices, qui ont lu attentivement chacun des textes et ont offert de précieuses suggestions aux auteur·e's des articles, que nous remercions également, pour leur généreuse contribution et leur agréable collaboration. 